\title{
Condensations Expelled from the Stars, and Pairs of Stars, Connected with Bright Filaments
}

\author{
A.L. Gyulbudaghian*1 and R.A. Mendezz ${ }^{\dagger 2}$ \\ ${ }^{1}$ Byurakan Astrophysical Observatory, Armenia, \\ ${ }^{2}$ Departamento de Astronomia, Universidad de Chile, Chile
}

\begin{abstract}
During several surveys of high-quality film-copies of the ESO B, R, ESO/SRC J, and EJ plates, except other young unstable objects (HH objects, star-forming regions, cometary nebulae) were found also two types of other unstable objects. 1. Bright condensations, connected with stars by bright filaments. 2. Pairs of stars, connected with each other by bright filaments. The objects of first type are mostly seen in infrared, on 2MASS K images. Due to their infrared colours, two of condensations and half of all stars can have thick dust discs.
\end{abstract}

Keywords: stars: star-forming regions, expelled condensations, pairs of stars

\section{Introduction}

During several surveys of Southern Hemisphere many types of interesting young nebular objects were found. These types are as follows. 1. Herbig-Haro objects. 2. Cometary nebulae. 3. Starforming regions. 4. Tight trapezium-type systems, consisting of young stars (Gyulbudaghian et al., 2004). 5. Radial systems of dark globules (Gyulbudaghian \& Mendez, 2015). 6. Saw-type ionized shock fronts (Gyulbudaghian, 2019a).We have done a systematic inspection of high-quality film-copies of the ESO B, R, ESO/SRC J, and EJ plates. We looked at all the Southern plate prints, but the final detailed search we concentrated mainly on places, where dark clouds could be easily identified on the prints.

In this paper we present the results of discovering (during the surveys) also of two other types of interesting objects. 1. Bright condensations, connected with stars by bright filaments (jets?). 2. Pairs of stars, connected by bright filaments. These two types of objects are very interesting and their further investigation will be very useful.

\section{Condensations, Expelled from the Stars}

1. Object CLN70 (Gyulbudaghian, 2011). This object on DSS2 R image is a pair of stars, connected with a filament (see Figure 1). Each star is connected with a condensation by a filament. The data on condensations are summarized in Table 1 (N1 and N2) and on stars in Table 2 (N1 and N2). We can see in Table 1, that condensation N1 has only R colour, hence it can be an HH object.

2. The star SAO 235903 (HD 70584, B8/9V). On DSS2 R image it is a bright nebular star (N3 in Table 2), on 2MASS K image it is a star with two condensations (N3 and N4 in Table 1) on one straight filament (the condensations are seen also on DSS2 R image). These images are presented in Figure 2.

3. Star-forming region SFR 2 (Gyulbudaghian, 2019a). This object is well seen on 2MASS K image. With the star (N4 in Table 2) two condensations (N5 and N6 in Table 1) are connected with bright filaments (see Figure 3).

\footnotetext{
*agyulb@bao.sci.am, Corresponding author

${ }^{\dagger}$ rmendez@u.uchile.cl
} 


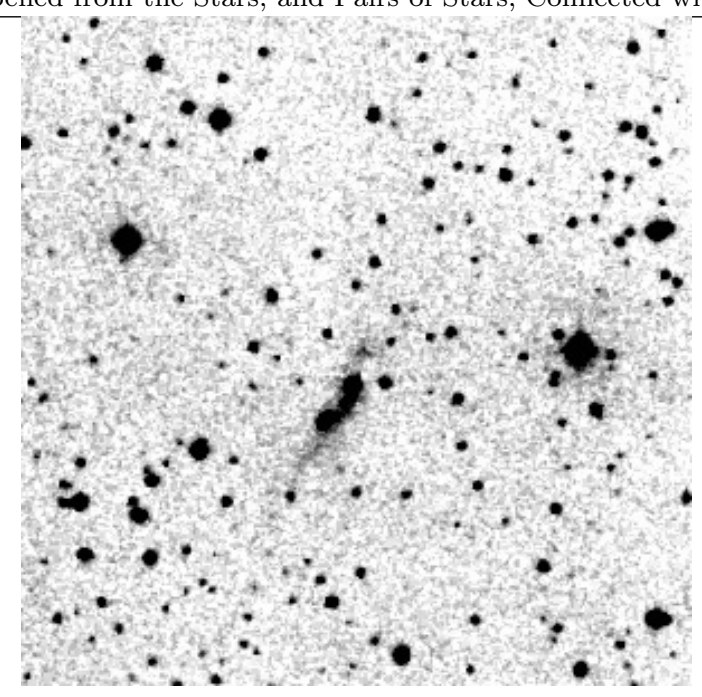

Figure 1. DSS2 R image of CLN70. The sizes of this and other images are $6^{\prime} \times 6^{\prime}$. For this and other images $\mathrm{N}$ is to the top, $\mathrm{E}$ is to the left.

Table 1. Data on condensations, connected with stars by filaments

\begin{tabular}{|c|c|c|c|c|c|c|c|c|c|}
\hline $\mathbf{N N}$ & $\alpha(\mathbf{2 0 0 0})$ & $\delta(\mathbf{2 0 0 0})$ & $\mathbf{B}$ & $\mathbf{B}-\mathbf{V}$ & $\mathbf{R}$ & $\mathbf{J}$ & $\mathbf{J}-\mathbf{H}$ & $\mathbf{H}-\mathbf{K}$ & $\mathbf{Q}$ \\
\hline \hline 1 & $06^{h} 00^{m} 04.72^{s}$ & $32^{\circ} 06^{\prime} 44^{*}$ & - & - & 18.200 & - & - & - & - \\
2 & 060007.76 & 320526 & 19.75 & 2.25 & 17.870 & 15.215 & 0.616 & 0.254 & 0.184 \\
3 & 082035.90 & -501859 & - & - & - & 13.836 & 0.757 & 0.311 & 0.228 \\
4 & 082036.54 & -501905 & - & - & 12.820 & 14.151 & 0.895 & 0.275 & 0.427 \\
5 & 085837.72 & -423211 & 20.84 & - & - & 16.077 & 1.048 & 0.462 & 0.263 \\
6 & 085837.72 & -423216 & - & - & - & 16.390 & 1.233 & 0.744 & -0.032 \\
7 & 103525.35 & -591448 & - & - & - & 13.583 & 0.853 & 0.384 & 0.200 \\
8 & 103525.64 & -591452 & - & - & - & 14.842 & 1.051 & 0.487 & 0.223 \\
9 & 103836.62 & -580238 & - & - & - & 13.657 & 0.456 & 0.227 & 0.070 \\
10 & 103836.85 & -580243 & - & $\mathrm{V}=16.690$ & - & 15.221 & 0.613 & 0.499 & -0.228 \\
11 & 150325.36 & -632320 & - & - & 19.670 & 14.353 & 1.582 & 0.889 & 0.071 \\
12 & 175833.86 & -260724 & - & - & - & 17.399 & 3.207 & 2.086 & -0.339 \\
\hline
\end{tabular}

4. Object vdBH 40b (van den Bergh \& Herbst, 1975). The star (N5 in Table 2, B2II) on DSS2 R image has a bright reflection nebula. On 2MASS K image there are two condensations (N7 and N8 in Table 1), connected with the star by a straight filament (see Figure 4).

5. The star SAO 238289 (HD 92383, B0.5 Vn, object N6 in Table 2) on DSS2 R image has a bright reflection nebula. On 2MASS K image two condensations (N9 and N10 in Table 1) are on the straight filament,connected with the star. This filament is seen also on DSS2 R image (see Figure 5).

6. The star DG Cir. The star (N7 in Table 2) on DSS2 R image is a single star, on 2MASS K image has a filament, with a condensation (object N11 in Table 1) at the end (see Figure 6).

7. Star-forming region HHL 59 (Gyulbudaghian \& Mendez, 2014). On the DSS2 R image there are two stars with a bright nebulosity, on the 2MASS K image there is a compact bright nebula (with embedded IR stars) with filaments and condensations at the ends of filaments. The star N8 (see Table 2) has a condensation N12 (see Table 1), connected with a filament (see Figure 7).

In Table 1 are summarized the data on condensations. In column 1 is presented the number of condensations, in columns 2 and 3 - their coordinates, in columns 4-9-the colours of stars, in column 10 - reddening free quantity $\mathrm{Q}=(\mathrm{J}-\mathrm{H})-1.70(\mathrm{H}-\mathrm{K})$. Values of $\mathrm{Q}<-0.10$ are indicative of an IR excess consistent with a dust disc (Comerón et al., 2005). It is possible to see from Table 1, that there are only two objects with $\mathrm{Q}<-0.10$ : objects N10 and N12. 
Table 2. Data on condensations, connected with stars by filaments

\begin{tabular}{|c|c|c|c|c|c|c|c|c|c|}
\hline NN & $\alpha(\mathbf{2 0 0 0})$ & $\delta(\mathbf{2 0 0 0})$ & $\mathbf{B}$ & $\mathbf{B - V}$ & $\mathbf{R}$ & $\mathbf{J}$ & $\mathbf{J}-\mathbf{H}$ & $\mathbf{H}-\mathbf{K}$ & $\mathbf{Q}$ \\
\hline \hline 1 & $06^{h} 00^{m} 05.15^{s}$ & $32^{\circ} 06^{\prime} 26^{\circ}$ & 15.030 & 0.450 & 13.60 & 13.235 & 0.941 & 2.143 & -2.702 \\
2 & 060006.35 & 320606 & 16.240 & 2.250 & 14.42 & 13.17 & 0.677 & 0.215 & 0.311 \\
3 & 082034.83 & -501850 & 9.354 & 0.298 & 9.05 & 8.276 & 0.047 & 0.275 & -0.217 \\
4 & 085239.51 & -423159 & - & - & 20.53 & 13.919 & 1.477 & 0.635 & 0.397 \\
5 & 103526.17 & -591459 & 10.624 & 0.356 & 10.04 & 9.11 & 0.079 & 0.17 & -0.21 \\
6 & 103836.37 & -580229 & 9.356 & 0.039 & 9.30 & 9.092 & 0.059 & 0.057 & -0.038 \\
7 & 150323.80 & -632259 & 13.630 & 0.500 & 12.42 & 9.75 & 1.004 & 0.924 & -0.587 \\
8 & 175834.09 & -260656 & 19.650 & 2.470 & 15.20 & 10.048 & 2.265 & 1.817 & -0.823 \\
\hline
\end{tabular}

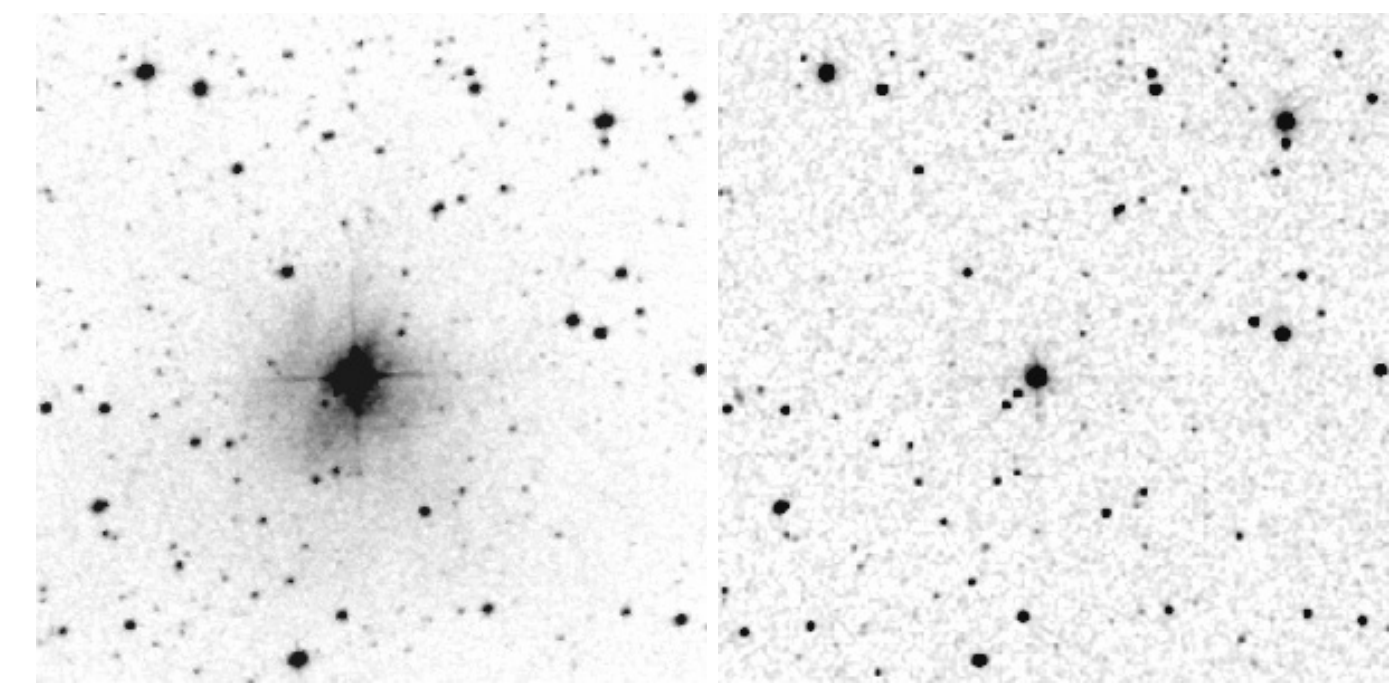

Figure 2. Left panel: DSS2 R image of the nebular star SAO 235903. Right panel: 2MASS K image of the nebular star SAO 235903.

\section{Stars, connected with bright filaments}

In Table 2 the data on stars, connected with condensations, are given. In column 1 is the number of stars, in columns 2 and 3 - their coordinates, in columns 4-9-the colours of stars, in column 10 - the reddening free quantity Q. From Table 2 it is possible to see, that there are 5 objects with $\mathrm{Q}<-0.10$. Hence, more than half of stars in the table can have dust discs.

1.Star-forming region CLN 70 (Gyulbudaghian, 2011). As is already mentioned above, on DSS2 R image there are two stars, connected with a bright filament (see Figure 1). Star N1 has a large negative value of $\mathrm{Q}$, hence it has a thick dust disc, but star $\mathrm{N} 2$ has a positive value of $\mathrm{Q}$, it has no dust disc (see Table 3).

2.Star-forming region SFR 3 (Gyulbudaghian, 2019a). On the DSS2 R image there is a pair of stars (N5 and N6 in Table 3, see Figure 8, left panel), connected with a pair of filaments. Both stars have large negative values of $\mathrm{Q}$, hence they have thick dust discs. On the 2MASS J image there is again a pair of stars (N3 and N4 in Table3), connected with a pair of filaments (see Figure 8, right panel). Both stars have positive values of $\mathrm{Q}$, they have no dust disc.

3.Two stars (N7 and N8 in Table 3), connected with a bright filament on the DSS2 B image (see Figure 9), in other colours the filament is absent. Both stars have positive values of $\mathrm{Q}$ - they have no dust disc.

4.Star-forming region HHL 59 (Gyulbudaghian \& Mendez, 2014). Two stars (N9 and N10 in Table 3), connected with a pair of filaments (better seen on the image, obtained with SII filter, see Figure 3, left panel in Gyulbudaghian \& Mendez (2014)). Both stars have large negative values of Q, hence they have thick dust discs.

In Table 3 the data on stars in pairs are given. In column 1 the number of stars is given, in columns 2 and 3 - their coordinates, in columns $4-9$ - the colours of stars, and in column 10 - the reddening 


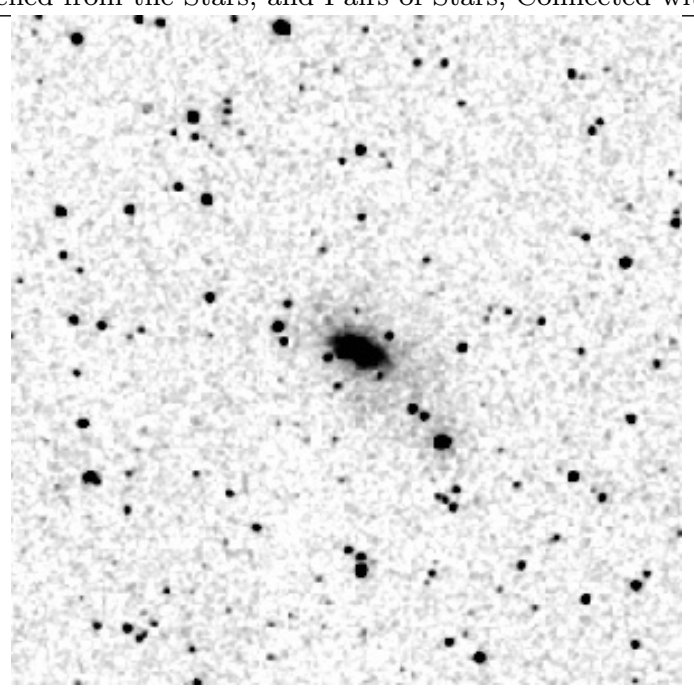

Figure 3. 2MASS K image of the star-forming region SFR 2.

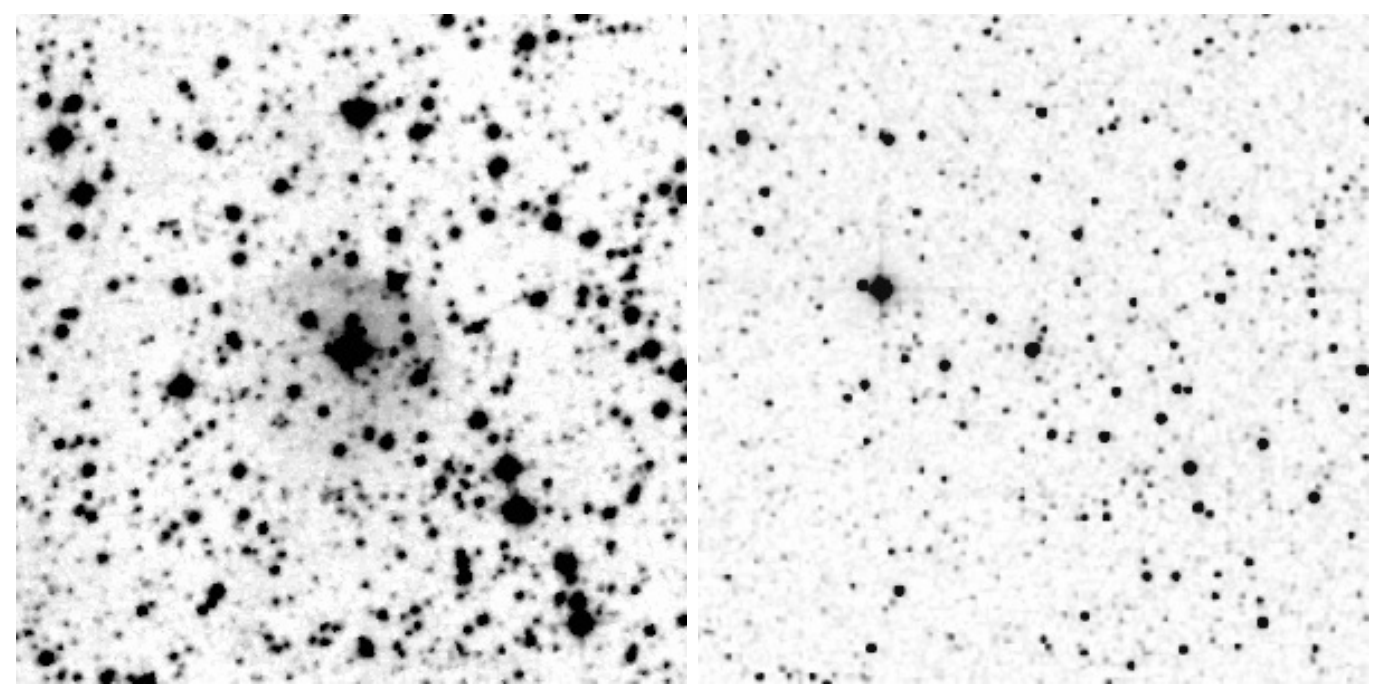

Figure 4. Left panel: DSS2 R image of vdBH 40b. Right panel: 2MASS K image of vdBH 40b.

free quantity $\mathrm{Q}$. We can see, that there are 5 objects with $\mathrm{Q}<-0.10$, hence half of the stars in the table can have dust discs.

\section{Conclusion}

As a result of searches on the high-quality film-copies of the plates of the Southern Hemisphere, except of several types of unstable objects, two other types of objects were also found. 1. Stars, connected with the condensation by a bright filament. Eight such objects were found, in four of them the stars are connected with two condensations, in others with one condensation. There is an opportunity, that the condensations were expelled from the stars. Almost all the condensations of these objects are seen only on the 2MASS K images. Two condensations and half of stars have infrared colours, typical for stars with thick discs. Three stars have spectral class B. 2. The pairs of stars, connected with each other by bright filaments. There are arch-shaped and also straight filaments. One of the filaments is seen only on DSS2 B image. Half of the stars have infrared colours, typical for stars with thick dust discs. 


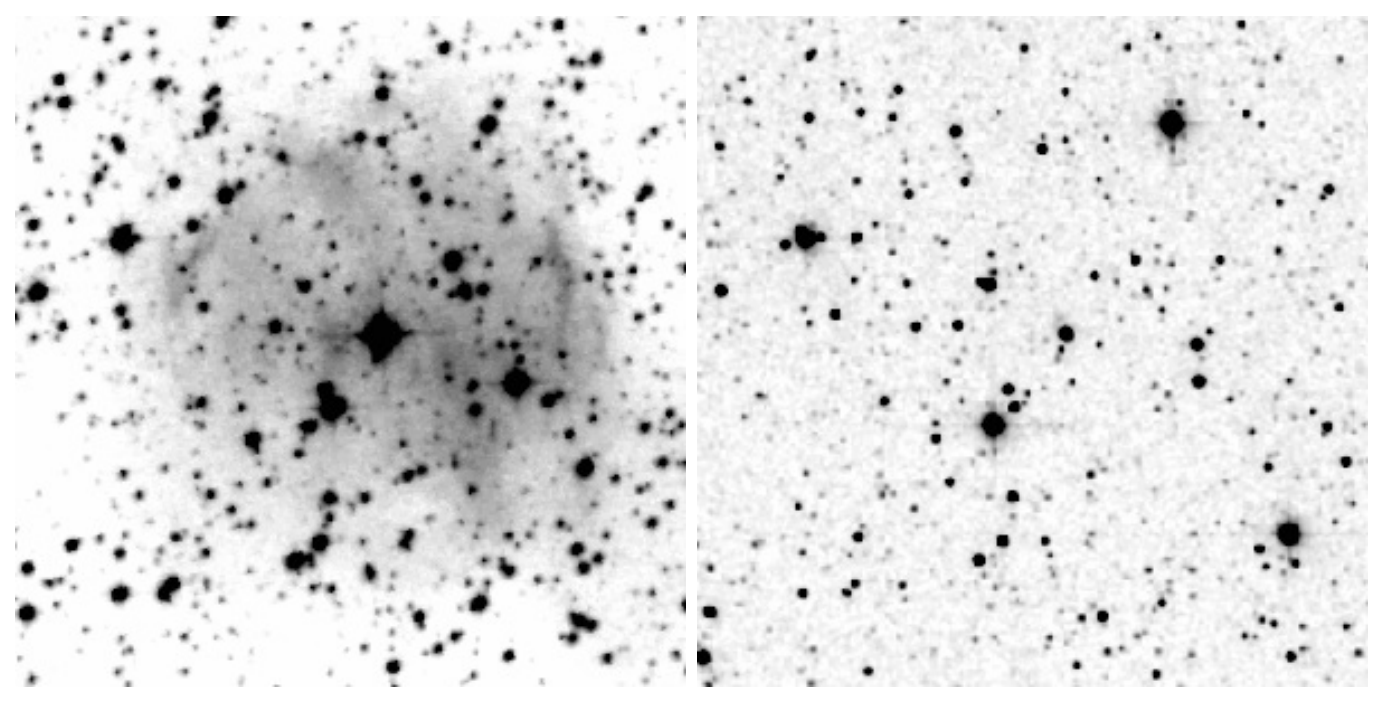

Figure 5. Left panel: DSS2 R image of the nebular star SAO 238289. Right panel: 2MASS K of the nebular star SAO 238289.

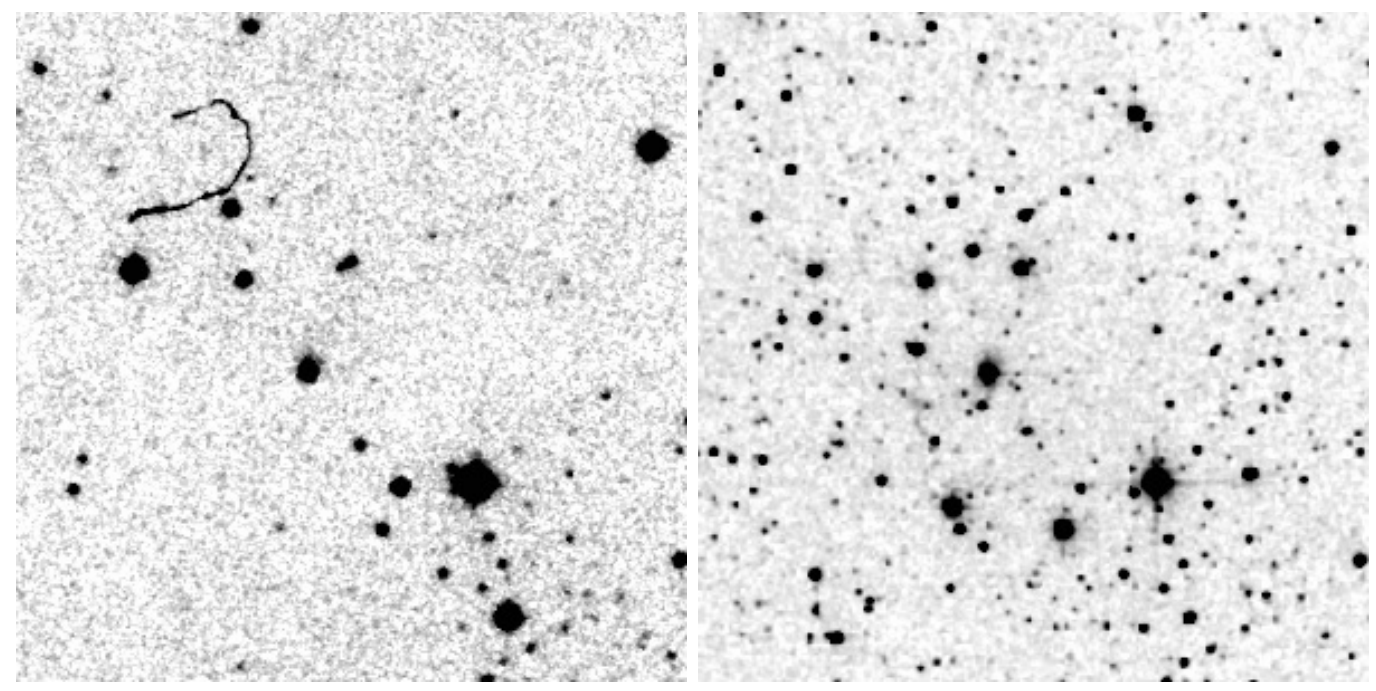

Figure 6. Left panel: DSS2 R image of the star DG Cir. Right panel: 2MASS K of the star DG Cir.

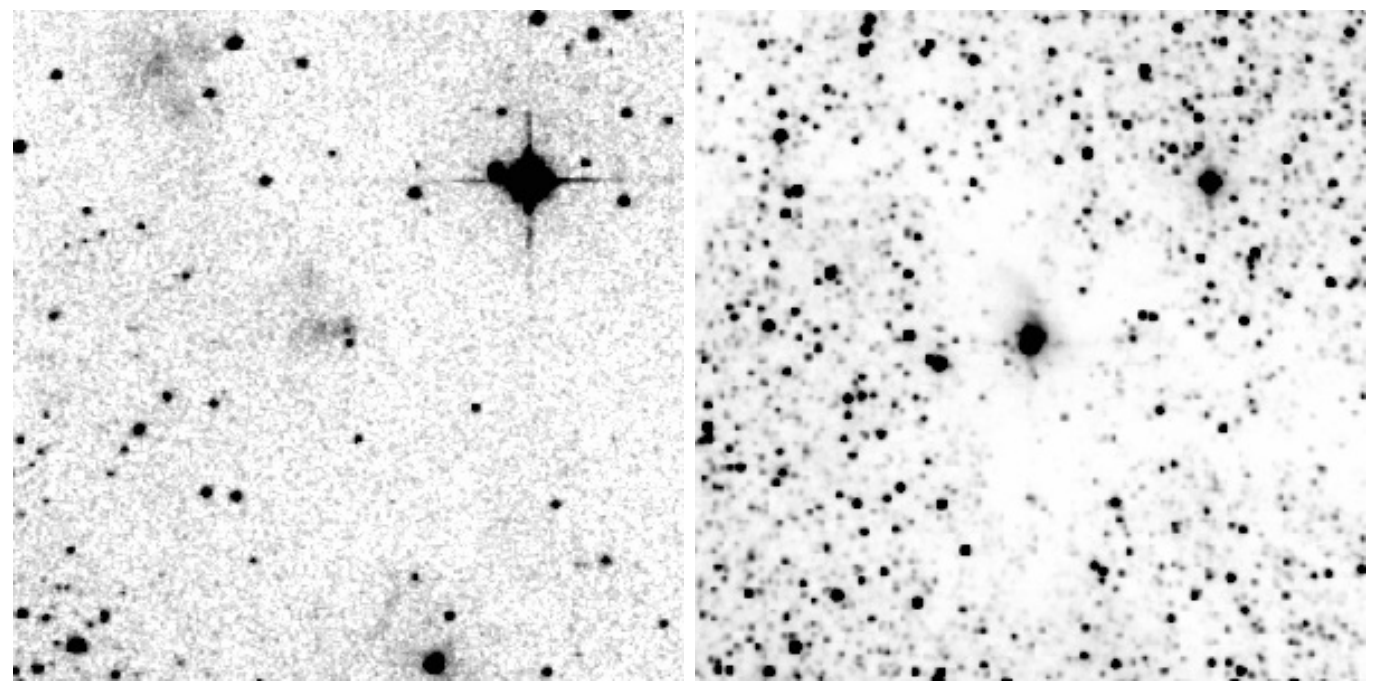

Figure 7. Left panel: DSS2 R image of the star-forming region HH L59. Right panel: 2MASS K of the star-forming region HH L59. 
Table 3. Data on the components of pairs of stars, in which the components are connected with each other by bright filaments

\begin{tabular}{|c|c|c|c|c|c|c|c|c|c|}
\hline NN & $\alpha(\mathbf{2 0 0 0})$ & $\delta(\mathbf{2 0 0 0})$ & $\mathbf{B}$ & $\mathbf{B - V}$ & $\mathbf{R}$ & $\mathbf{J}$ & $\mathbf{J}-\mathbf{H}$ & $\mathbf{H}-\mathbf{K}$ & $\mathbf{Q}$ \\
\hline \hline 1 & $06^{h} 00^{m} 05.15^{s}$ & $32^{\circ} 06^{\prime} 26^{*}$ & 15.03 & 0.45 & 13.6 & 13.235 & 0.941 & 2.143 & -2.702 \\
2 & 060006.35 & 320606 & 16.24 & 2.25 & 14.42 & 13.17 & 0.677 & 0.215 & 0.311 \\
3 & 082251.67 & -420749 & - & - & - & 14.576 & 1.955 & 1.516 & -0.622 \\
4 & 082251.91 & -420803 & - & - & - & 14.342 & 0.893 & 1.591 & -1.812 \\
5 & 082252.84 & -420730 & 16.28 & -0.8 & 14.72 & 13.17 & 0.857 & 0.496 & 0.014 \\
6 & 082252.84 & -420746 & 16.64 & 0.32 & 15.08 & 14.939 & 1.449 & -0.369 & 2.076 \\
7 & 082737.36 & -510950 & 19.83 & - & 17.13 & 13.48 & 1.02 & 0.435 & 0.28 \\
8 & 082739.51 & -510645 & 16.01 & 1.12 & 14.27 & 13.063 & 0.474 & 0.149 & 0.221 \\
9 & 175834.09 & -260656 & 19.65 & 2.47 & 15.2 & 10.042 & 2.265 & 1.817 & -0.823 \\
10 & 175834.23 & -260651 & 16.37 & -0.54 & 12.48 & 11.476 & 1.942 & 1.439 & -0.504 \\
\hline
\end{tabular}

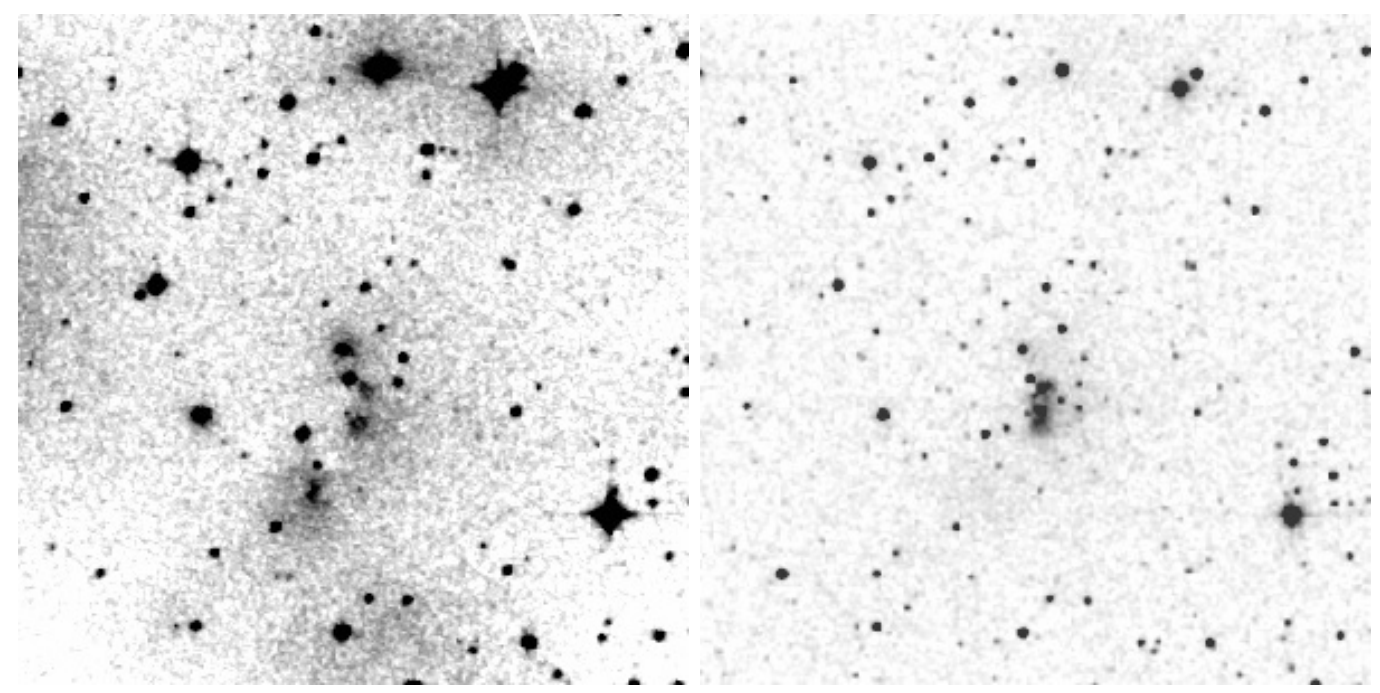

Figure 8. Left panel: DSS2 R image of the star-forming region SFR 3. Right panel: 2MASS K of the star-forming region SFR 3.

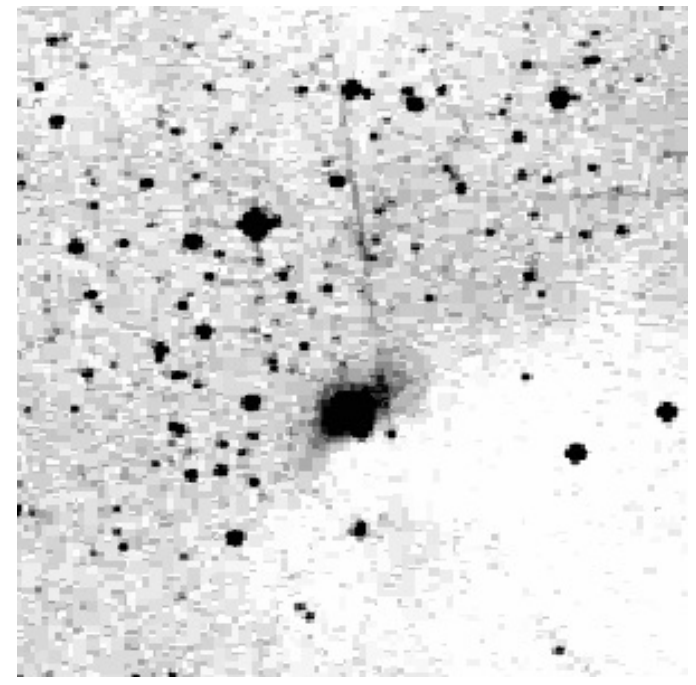

Figure 9. DSS2 B image of two stars, connected with a filament. 


\section{References}

Comerón F., Schneider N., Russeil D., 2005, Astron. Astrophys. , 433, 955

Gyulbudaghian A. L., 2011, Astrophysics, 54, 476

Gyulbudaghian A. L., 2019a, Astrophysics, 62, 360

Gyulbudaghian A. L., 2019b, Communications of the Byurakan Astrophysical Observatory, 66, 20

Gyulbudaghian A. L., Mendez R. A., 2013, Astrophysics, 56, 531

Gyulbudaghian A. L., Mendez R. A., 2014, Astrophysics, 57, 520

Gyulbudaghian A. L., Mendez R. A., 2015, Rev. Mex. Astron. Astrofis., 51, 183

Gyulbudaghian A. L., May J., González L., Méndez R. A., 2004, Rev. Mex. Astron. Astrofis. , 40, 137

van den Bergh S., Herbst W., 1975, Astron. J. , 80, 208 